\title{
Control of expression of the ICE R391 encoded UV-inducible cell-sensitising function
}

\author{
Patricia Armshaw ${ }^{\dagger}$ and J Tony Pembroke ${ }^{* \dagger}$
}

\begin{abstract}
Background: Many SXT/R391-like enterobacterial Integrative Conjugative Elements (ICEs) have been found to express an atypical, recA-dependent, UV-inducible, cell-sensitising phenotype observed as a reduction in postirradiation cell survival rates in host cells. Characterisation of a complete deletion library of the prototype ICE R391 identified the involvement of three core ICE genes, orfs90/91 encoding a putative transcriptional enhancer complex, and orf43, encoding a putative type IV secretion system, outer membrane-associated, conjugative transfer protein.

Results: In this study, expression analysis of orf43 indicated that it was up-regulated as a result of UV irradiation in an orfs90/91-dependent manner. Induced expression was found to be controlled from a site preceding the gene which required functional orfs90/91. Expression of orfs90/91 was in turn found to be regulated by orf96, a $\lambda$ cl-like regulator. Targeted construction of ICE R391 deletions, RT-PCR and qRT-PCR analysis confirmed a regulatory link between orfs90/91 and orf43 while site-directed mutagenesis of orf43 suggested an association with the cell membrane was a prerequisite for the cytotoxic effect.

Conclusions: Because of the recA-dependence of the effect, we hypothesise that UV induction of RecA results in cleavage of the cl-like ICE-encoded repressor protein, the product of orf96. This in turn allows expression of the transcriptional enhancer complex encoded by orfs90/91, which we conclude stimulates transcription of orf43, whose product is directly responsible for the effect.
\end{abstract}

Keywords: SXT/R391 ICE, Type IV secretion system, Conjugative transfer, UV-induced sensitisation and cytotoxicity

\section{Background}

Integrative Conjugative Elements (ICEs) are a class of bacterial mobile genetic elements that encode features necessary for their site-specific integration and excision from host genomes, self-circularisation and transfer by conjugation [1,2]. ICEs are divided into families based on similarity between core genes (specifically the integrase gene) and the site of integration they utilise within host chromosomes. The SXT/R391 family share a highly similar integrase gene and integrate into the $\operatorname{prfC}$ gene of enterobacterial hosts [1,3]. In addition to encoding host beneficial traits such as antibiotic resistance determinants [3-5], many SXT/R391 family ICEs express an unusual cell-sensitising function [6-8]. Preliminary characterisation of the UV-inducible, cell-sensitising function of the prototype, ICE R391, determined the effect to

\footnotetext{
* Correspondence: tony.pembroke@ul.ie

${ }^{\dagger}$ Equal contributors

Molecular and Structural Biochemistry Laboratory, Department of Chemical and Environmental Sciences, University of Limerick, Limerick, Ireland
}

be $\operatorname{rec} A$-dependent [6], while further analysis based on construction of a deletion library of ICE R391 found that three core ICE genes, namely orfs $90 / 91$ and orf 43 were involved [8]. Deletion analysis also revealed that orf 96 , which encodes a putative $\lambda$ cI-like repressor protein [9], could only be deleted in strains where orfs $90 / 91$ had previously been removed suggesting that the repressor protein may prevent lethal expression of orfs 90/91. Additionally, cloning and controlled expression of both orfs $90 / 91$ and orf 43 revealed that expression of orf 43 alone was cytotoxic to wild type E. coli while expression of orfs $90 / 91$ was only cytotoxic to wild type $E$. coli cells harbouring the ICE R391. This indicated that orf43 was responsible for the observed UV-inducible cytotoxicity [8].

RecA is a well-documented regulatory protein involved in UV-induced proteolysis of repressor proteins associated with the SOS response [10]. Induction of RecA (some 50 fold) following UV irradiation, results in cleavage of phage $\lambda$ and phage $\lambda$-like cI repressors resulting in phage induction and indeed cleavage of other SOS repressors [10-13]. 
Bioinformatic analysis of the ICE R391 encoded orf96 has shown it encodes a cI-like repressor protein with homology to phage $\lambda^{434} \mathrm{cI}$ [9], while analysis of the ICE R391-encoded orfs $90 / 91$ has indicated that these genes may act as a putative transcriptional enhancer complex. It has been demonstrated that orfs $90 / 91$ stimulate the expression of ICE specific genes such as orf4 (jef, Figure 1) [14], which is an element-encoded excisionase, resulting in formation of increased levels of a circular form of the ICE, presumably as a transfer intermediate. The ICE R391encoded orf43, has previously been found to be essential for ICE R391 transfer [8]. Indeed, based on bioinformatic homology, orf43 is predicted to encode a putative TraV homolog, an outer membrane protein involved in the ICE type IV secretion system and thought to function in the construction and stabilisation of the outer-membrane portion of the mating pore required for ICE transfer by conjugation [15]. Deletion of the ICE R391-encoded orf43 was recently shown to abolish the UV-inducible sensitising effect of this ICE while clones expressing orf 43 under arabinose control were shown to compliment for the transfer deficiency but additionally mimic the cell toxicity associated with UV induction [8].
Here, a model is proposed (Figure 1) for the control of this unusual ICE R391 UV-inducible sensitising effect based on expression data examining the key genes involved and supported by a number of directed ICE R391 deletions.

\section{Results and discussion}

orfs90/91 stimulate orf43 transcription after exposure to

\section{UV irradiation}

We previously demonstrated that over-expression of orf 43 when cloned into the arabinose inducible pBAD33-orf 43 construct was responsible for the UV-inducible sensitisation observed in ICE R391 and other ICEs of the SXT/ R391 family [8]. Mutagenesis data also suggested that the putative transcriptional controller encoded by orfs $90 / 91$ was also involved, although not directly. To investigate the relationship between orfs90/91 and orf43, we utilised both qualitative and quantitative RT-PCR targeting these genes in different mutant backgrounds and with and without UV irradiation.

qRT-PCR analysis of expression levels pre and post exposure to $40 \mathrm{~J} \cdot \mathrm{m}^{-2} \mathrm{UV}$-irradiation indicated that orfs $90 / 91$, orf 43 and the previously documented UV-inducible orf4

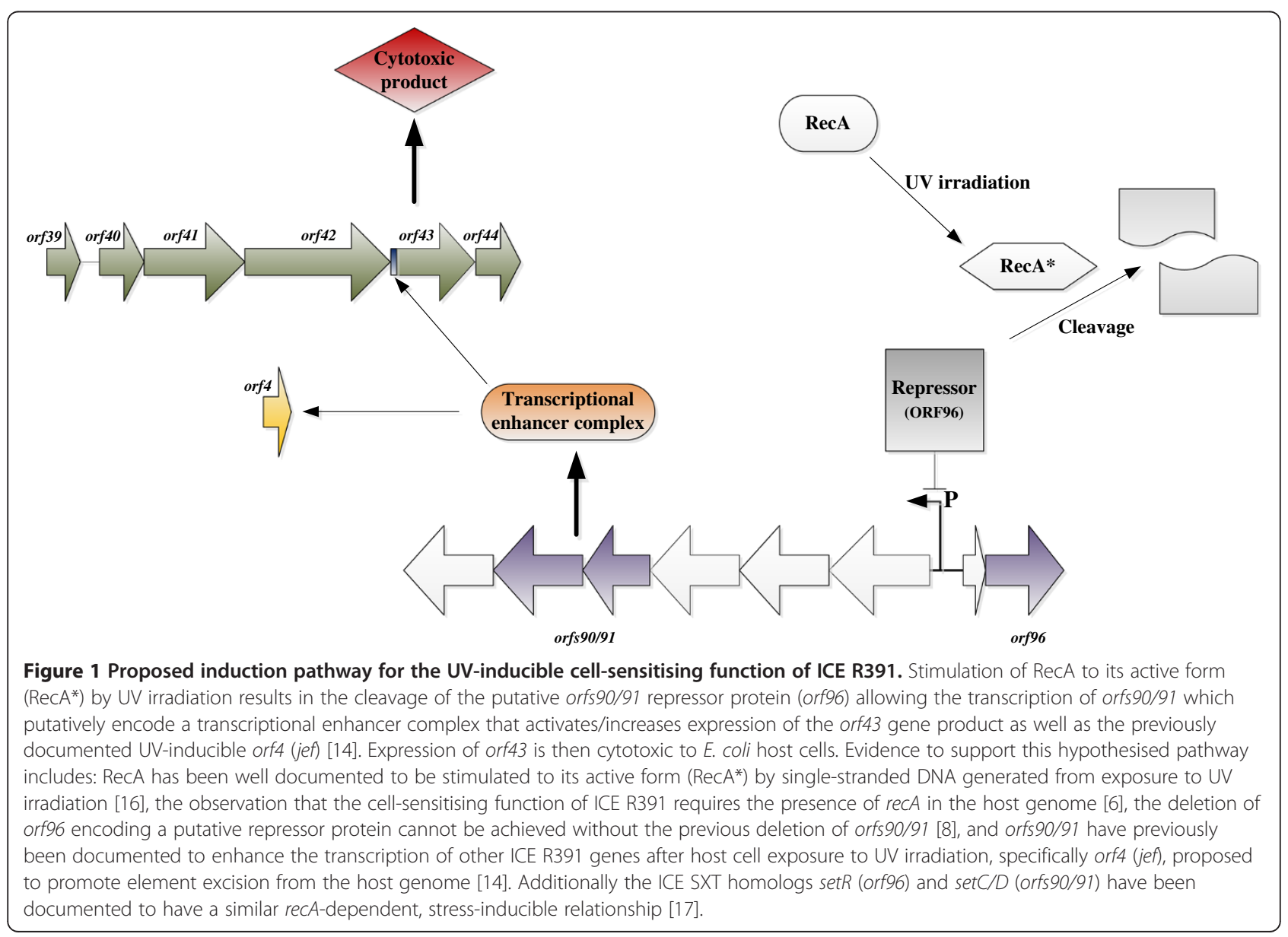


(jef, Figure 1) [14] were up-regulated after exposure to UV irradiation. Analysis indicated that orf4 (jef) specific mRNA levels were up-regulated 0.78 fold, orf43, 0.513 fold and orfs9091, 0.339 fold. In contrast other ICE R391 genes not involved in cell sensitisation [8] were not up-regulated post exposure: aph (encoding Kanamycin resistance) was down-regulated 0.23 fold post-exposure while orf31 (encoding a putative Lon protease) was also downregulated 0.19 fold post-exposure. Analysis of the upregulated genes in mutant backgrounds indicated that in a $\Delta$ orfs $90 / 91(\Delta 26)$ background, orf43 up-regulation was abolished (Figure 2) while analysis of orfs $90 / 91$ transcription in a $\Delta$ orf43 $(\Delta 14)$ background did not prevent $\operatorname{orf} 590 / 91$ specific mRNA up-regulation following UV irradiation (orfs90/91 up-regulated 0.61 fold in AB1157 R391 $\Delta 14)$. This indicated a dependency on orfs90/91 for orf43 up-regulation but not vice versa. Further analysis of orf43 transcription in a $\Delta$ orfs $40 / 41$ mutant $(\Delta 11)$ [8] demonstrated that deletion of these genes, upstream of orf43, did not prevent the UVinduced up-regulation of orf 43 mRNA, suggesting that inducible orf43 transcription was stimulated through a region directly in front of the orf 43 gene (Figure 2) and that this region should be investigated further. This observation is supported by previous deletion analysis where $\operatorname{orfs} 40 / 41(\Delta 11)$ and $\Delta \operatorname{orf} 42(\Delta 13)$ were deleted but retained the UV-inducible sensitising phenotype [8].

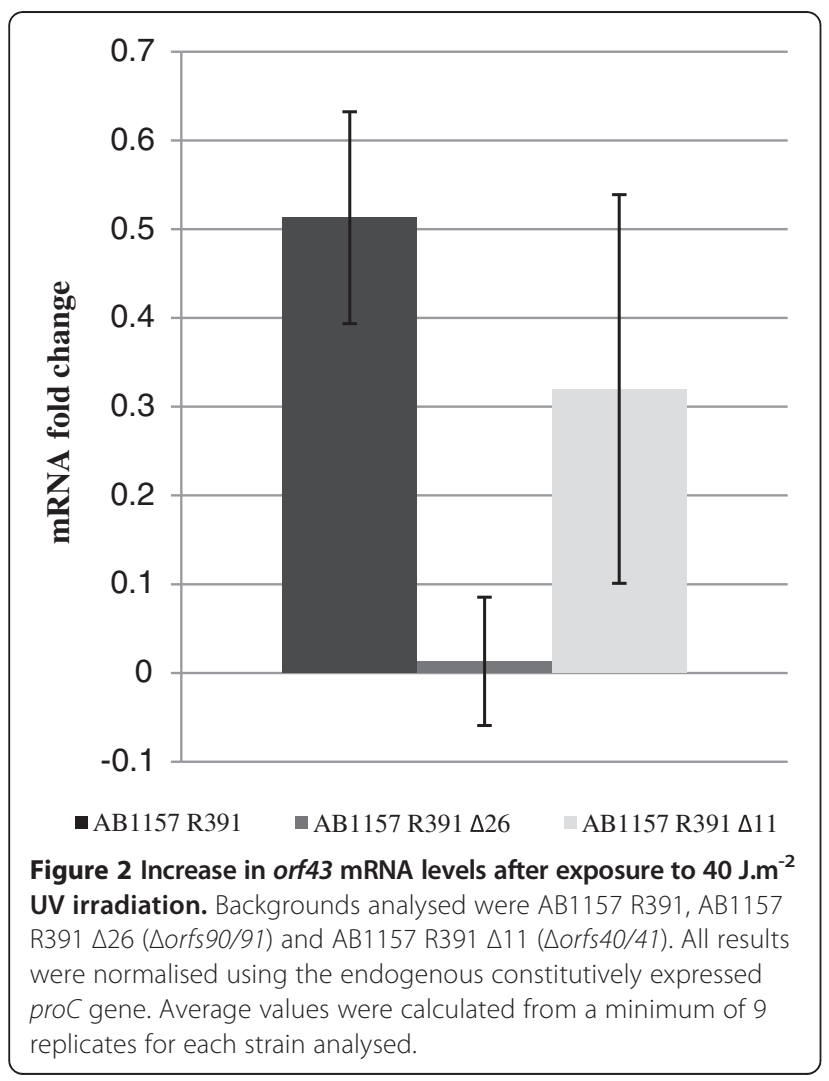

Analysis of the up-regulated orfs $90 / 91$ and orf43 mRNA decay rate post-exposure (Figure 3) revealed that orfs $90 /$ 91 mRNA levels were maximally up-regulated directly after exposure and decayed rapidly with a return to basal levels within 5 minutes post-exposure. However orf43 mRNA levels were maximally up-regulated 7 minutes post-exposure and up-regulated levels were sustained for a longer period of time, minimally over 30 minutes (Figure 3). The observation of the rapid increase and decay of $\operatorname{orfs} 90 / 91$ specific mRNA levels followed by a slower and longer sustained increase in orf43 specific mRNA levels supports the hypothesis that UV irradiation acts as an inducing agent for $\operatorname{orfs} 90 / 91$, which subsequently up-regulates the transcription of orf 43 possibly from a site preceding the gene.

\section{Cytotoxic orf43 transcription is regulated through a region directly upstream of orf43}

Based on previous observations with the $\Delta 11$ and $\Delta 13$ ICE R391 deletions, which deleted orfs40 to most of orf42 inclusive [8], the most likely location for an orf43 control site would be the last 36 bp specific to orf 42 directly in front of orf43. Comparative bioinformatic analysis of this region and the previously documented orfs $90 / 91$ regulated orf4 (jef) [14] uncovered a short 7 bp homologous DNA sequence (5'-AGAAGAT-3') present in front of both genes. This conserved sequence was located 77 bp upstream of orf4 (jef) but directly in front of orf43 where the last 2 base pairs of the sequence overlapped the first two base pairs of the predicted start codon of orf43. As no other recognisable promoter or operator region was predicted upstream of orf43, this 7 bp sequence may possibly represent a binding motif for the putative transcriptional enhancer (orfs90/91). However it is well known that transcriptional enhancer control sites can be difficult to predict [18] as they tend to be short DNA sequences lacking high sequence conservation even between enhancer types. To examine if the last $36 \mathrm{bp}$ specific to orf 42 and preceding orf43 did in fact contain a control site for orf43 transcription, orf43 specific mRNA expression was analysed in a number of specific deletion backgrounds spanning this putative control region [Table 1, Figure 4C]. Three directed ICE R391 deletion mutants were generated [Figure 4C] in an E. coli (AB1157 R391) background; the KOA deletion removed the genes orf32 to orf 42 and placed the inserted ampicillin cassette on the reverse complement to ensure removal of all possible promoters of orf 43 transcription except for the 36 bp directly in front of orf43, the $\mathrm{KOB}$ deletion removed the genes orf32 to orf42 similar to KOA but additionally removed the 36 bp directly in front of orf 43 while the KOC deletion was identical to the KOA mutation, preserving the putative 36 bp control site but also contained an additional secondary zeocin resistant deletion which 


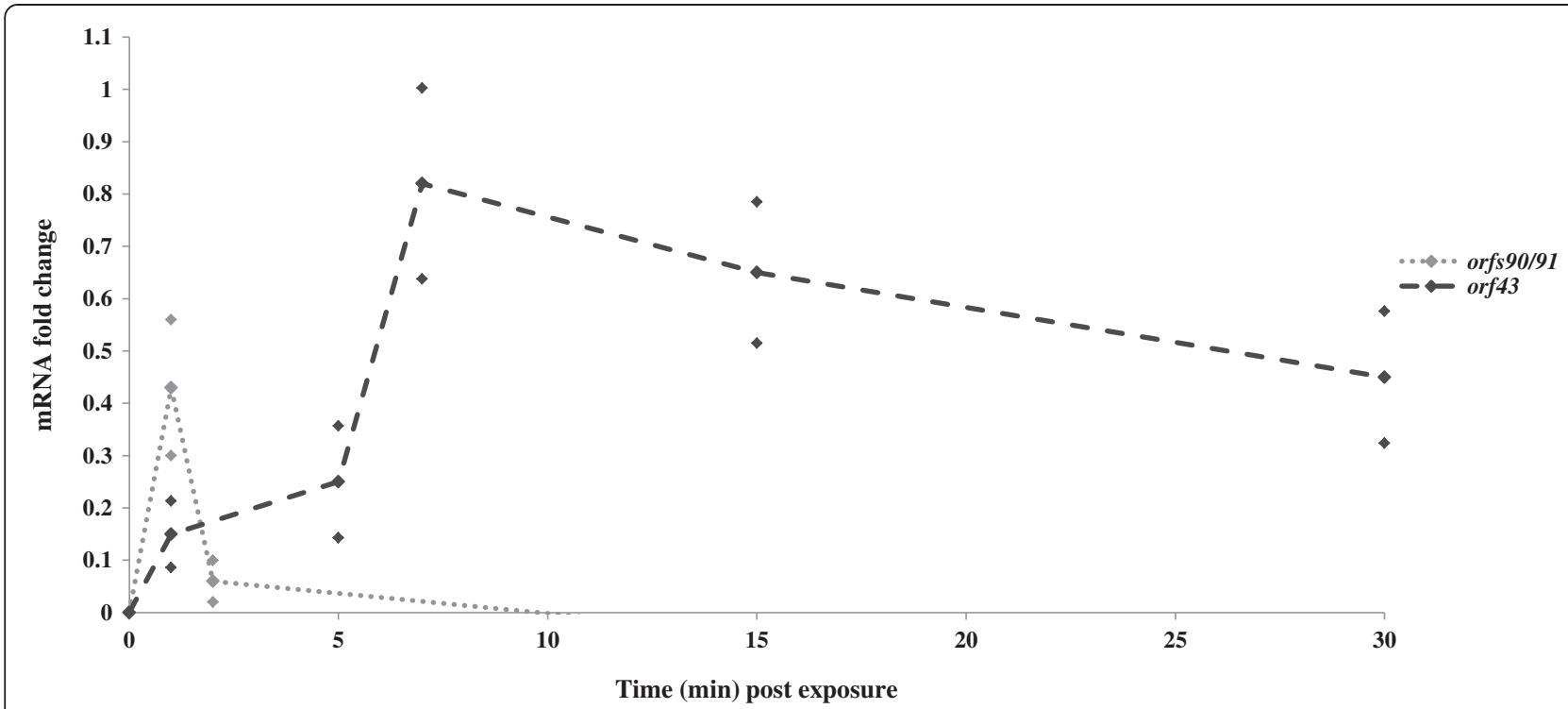

Figure 3 Decay of AB1157 R391 orfs90/91 and orf43 mRNA levels after exposure to $40 \mathrm{~J} . \mathrm{m}^{-2}$ UV irradiation. All results were normalised using the endogenous constitutively expressed proC gene. Standard deviation is denoted by markers above and below all data points. orfs $90 / 91$ specific mRNA levels were up-regulated immediately post exposure to UV irradiation but quickly decayed. orf43 specific mRNA levels were maximally up-regulated 7 minutes post exposure and elevated levels were sustained for over 30 minutes post exposure.

removed orfs 90/91. These three deletion mutations were screened by both qualitative and quantitative UV survival assays to determine their effect on the cell-sensitising function [Figure $4 \mathrm{~A}$ ] and additionally were examined by RT-PCR to determine if orf43 specific mRNA transcription still occurred [Figure 4B]. The KOA mutant retained the UV-inducible sensitising function [Figure 4A] and orf 43 mRNA transcription [Figure $4 \mathrm{~B}$ ], while the $\mathrm{KOB}$ and KOC mutations abolished the sensitising function as well as orf43 mRNA transcription. As the KOB and KOC mutations deleted either the proposed orfs $90 / 91$ control site or orfs90/91 genes respectively and both of these deletions prevented cytotoxic orf 43 transcription, this analysis supported the hypothesis that orfs $90 / 91$ encode a transcriptional enhancer complex that activates cytotoxic orf 43 transcription through the 36 bp sequence directly in front of orf43. In addition, cloning of orf43 with the predicted control site in front of the gene showed that the cytotoxic function could be repressed only in cells not containing orfs90/91 (data not shown), again supporting the hypothesis.

\section{Site-directed mutagenesis of Orf43}

Bioinformatic analysis of orf43 indicated that it belongs to a highly conserved TraV-like family of transfer proteins involved in type IV secretion systems required for conjugation [8]. Site-directed mutagenesis of pBAD33-orf43 was carried out to convert two leucines at a.a. positions 47 and 48 to prolines in the predicted Orf43 protein (GenBank: AAM08037). Insertion of two prolines was expected to disrupt the $\alpha$-helical transmembrane spanning region of
Orf43 by creating a $30^{\circ}$ bend [19]. This mutation was found to cause loss of the cytotoxic function of pBAD33orf43 [8] as there was no observable decline in host cell growth rates after induction of the mutant clone compared to the wild type clone [Figure 5A,B]. Since introduction of membrane disruptive mutations abolish the effect, this is suggestive that membrane association is required in addition to over-expression of the Orf43 protein for sensitisation and cytotoxicity associated with this ICE product. Other directed mutations such as conversion of glutamine at position 115 (predicted in the periplasmic region of Orf43) to asparagine (Q115 to N115) was shown to have no effect on UV sensitisation or orf43 transcription [Figure 5C,D].

\section{Conclusions}

\section{Hierarchical control of the ICE R391 UV-inducible sensitising effect}

Many SXT/R391-like ICEs reduce post UV survival rates of $E$. coli host cells through the action of a recA-dependent process $[6,20]$. Mutational analysis of the ICE R391 determined that the core genes orfs $90 / 91$ and orf 43 were required for expression of the cell-sensitising function [8] while bioinformatic analysis indicated that orf96 likely encodes a $\lambda$ cI-like repressor similar to RecA substrates in other phage systems that are cleaved following SOS induction [9]. Initial attempts to delete orf 96 proved fruitless and no deletion could be isolated. However a $\Delta$ orf 96 $(\Delta 28)$ deletion [8] could be isolated in an $\Delta o r f s 90 / 91$ mutant background suggesting that orf 96 may control expression of orfs90/91 which we have shown here directly 
Table 1 Genotype of bacterial strains, plasmids and ICE R391 mutants used

\begin{tabular}{|c|c|c|}
\hline Strain & Genotype & Source \\
\hline AB1157 & 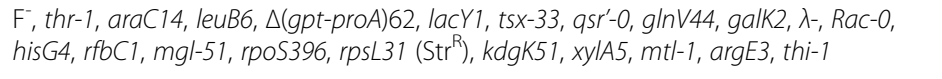 & $\begin{array}{l}\text { E. coli genetic stock centre (CGSC), Yale } \\
\text { University, New Haven, Connecticut, USA }\end{array}$ \\
\hline TOP10 & 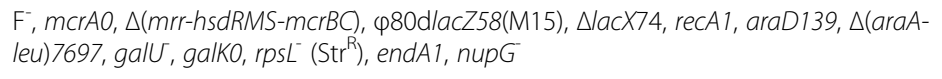 & Bio-Sciences, Dun Laoghaire, Dublin, Ireland \\
\hline P125109 & S. Enteritidis PT4 wild type (NCTC 13349), Nal ${ }^{R}$ & $\begin{array}{l}\text { National Collection of Type Cultures (NCTC), } \\
\text { Salisbury, UK }\end{array}$ \\
\hline Plasmid & Genotype & Source \\
\hline pBAD33-orf43 & $\mathrm{Cm}^{\mathrm{R}}$, p15A ori, $\mathrm{P}_{\mathrm{BAD}} \mathrm{L}$-arabinose inducible, orf 43 & Armshaw and Pembroke, 2013 [8] \\
\hline $\begin{array}{l}\text { pBAD33-orf43 } \\
\text { [SM12] }\end{array}$ & $\begin{array}{l}\mathrm{Cm}^{\mathrm{R}} \text {, p15A ori, } \mathrm{P}_{\mathrm{BAD}} \mathrm{L} \text {-arabinose inducible, orf } 43 \text { containing mutation converting } \\
\text { two leucines to prolines at a.a. position } 47 \text { and } 48 .\end{array}$ & This study \\
\hline $\begin{array}{l}\text { pBAD33-orf43 } \\
\text { [SM56] }\end{array}$ & $\begin{array}{l}\mathrm{Cm}^{R}, \text { p15A ori, } P_{B A D} L \text {-arabinose inducible, orf } 43 \text { containing mutation converting } \\
\text { glutamine at position } 115 \text { to asparagine. }\end{array}$ & This study \\
\hline PKOBEG & $\mathrm{T}_{\mathrm{S}}, \mathrm{P}_{\mathrm{BAD}}$-gam-bet-exo cat $\left(\mathrm{Cm}^{\mathrm{R}}\right)$ & $\begin{array}{l}\text { Dr. P. Latour-Lambert, Institut Pasteur, } 25 \text { rue } \\
\text { du Dr Roux, Paris, France }\end{array}$ \\
\hline pUC18 & $\mathrm{Am}^{R}$ template for deletion mutant construction & Sigma-Aldrich, Arklow, Wicklow, Ireland \\
\hline pcDNA3. $1^{(+)}$ & $Z^{R}$ template for deletion mutant construction & $\begin{array}{l}\text { Invitrogen, Bio-Sciences, Dun Laoghaire, } \\
\text { Dublin, Ireland }\end{array}$ \\
\hline ICE & Genotype & Source \\
\hline R391 & $\mathrm{Km}^{\mathrm{R}}, \mathrm{Hg}^{\mathrm{R}}$ & $\begin{array}{l}\text { Dr R.W. Hedges, Royal Postgraduate Medical } \\
\text { School, London, UK }\end{array}$ \\
\hline R391 Mutant & Genotype & Source \\
\hline $\begin{array}{l}\text { AB1157 R391 } \\
\Delta 14(\Delta o r f 43)\end{array}$ & ICE R391 orf43 deletion strain, $\mathrm{Am}^{\mathrm{R}}$, UV, tra & Armshaw and Pembroke, 2013 [8] \\
\hline $\begin{array}{l}\text { AB1157 R391 } \\
\triangle 26(\Delta \text { orfs90/91) }\end{array}$ & ICE R391 orfs90/91 deletion strain, $\mathrm{Am}^{\mathrm{R}}$, UV, tra' & Armshaw and Pembroke, 2013 [8] \\
\hline $\begin{array}{l}\text { AB1157 R391 } \\
\Delta 11(\Delta \text { orfs40/41) }\end{array}$ & ICE R391 orfs40/41 deletion strain, $\mathrm{Am}^{\mathrm{R}}$, tra- & Armshaw and Pembroke, 2013 [8] \\
\hline $\begin{array}{l}\text { AB1157 R391 } \\
\triangle 25_{A m}^{R} \Delta 14_{Z e}^{R}\end{array}$ & ICE R391 orf90 - orf94 and orf43 deletion strain, $\mathrm{Am}^{\mathrm{R}}, \mathrm{Ze}^{\mathrm{R}}, \mathrm{UV}$, tra ${ }^{-}$ & This study \\
\hline $\begin{array}{l}\text { AB1157 R391 } \\
\text { KOA }\end{array}$ & ICE R391 orf32 - orf42 (29575 bp - 41491 bp) deletion strain, $\mathrm{Am}^{\mathrm{R}}$, tra ${ }^{-}$ & This study \\
\hline $\begin{array}{l}\text { AB1157 R391 } \\
\text { KOB }\end{array}$ & ICE R391 orf32 - orf42 (29575 bp - 41527 bp) deletion strain, Am ${ }^{\mathrm{R}}$, UV, tra- & This study \\
\hline $\begin{array}{l}\text { AB1157 R391 } \\
\text { KOC }\end{array}$ & $\begin{array}{l}\text { ICE R391 orf32 - orf42 (29575 bp - } 41491 \text { bp) and orfs90/91 deletion strain, Am }{ }^{R} \text {, } \\
Z^{R} e^{R}, U^{\prime} \text {, tra }\end{array}$ & This study \\
\hline
\end{tabular}

$\mathrm{Str}^{\mathrm{R}}$ is streptomycin resistant; $\mathrm{Cm}^{\mathrm{R}}$ is chloramphenicol resistant; $\mathrm{Km}^{\mathrm{R}}$ is kanamycin resistant; $\mathrm{Hg}^{\mathrm{R}}$ is mercury resistant; Ze ${ }^{\mathrm{R}}$ is zeocin resistant; $\mathrm{T}_{\mathrm{s}}$ is temperature sensitive; $\mathrm{Nal}^{\mathrm{R}}$ is nalidixic acid resistant and $\mathrm{Am}^{\mathrm{R}}$ is ampicillin resistant. UV is UV-inducible cell-sensitising function abolished; tra is conjugative transfer deficient mutant.

control expression of orf43, the ultimate instigator of the cytotoxicity associated with ICE R391. The data presented here and in Armshaw and Pembroke (2013) [8] have led to the development of a model to explain the control of UV-inducible sensitisation (Figure 1). We hypothesise that UV irradiation of $E$. coli induces the host RecA protein which results in cleavage of the ICE R391 encoded product of orf 96 , the phage $\lambda^{434}$ cI-like ICE repressor. We propose that cleavage of Orf96 in turn leads to expression of orfs $90 / 91$ which in turn leads to up-regulation of orf 43 and other ICE R391 genes such as orf4 (jef) [14]. We have previously demonstrated that up-regulation of orf4 (jef) leads to increased ICE R391 transfer [14]. In the related ICE SXT, Beaber et al., (2004) [17] demonstrated that
SetR, the SXT homolog of Orf96, acted as a repressor of ICE SXT transfer and that it is bound to ICE operators that controlled setC/D, SXT homologs of orfs $90 / 91$, in a similar way to our proposal for ICE R391. They also proposed that repression was lifted by induced RecA protein cleaving the SetR repressor in a similar manner to our proposal for orfs $90 / 91$. The recA dependence for the ICE R391 UV-sensitising effect [6], the similarity to the SXT system [17], the deletion data and qRT-PCR data presented here support the model presented.

It would thus appear that UV irradiation is the instigator of the control loop leading to over expression of orf43 which leads to cytotoxicity. Since normal levels of Orf43 are required for ICE transfer and play a key part in 


\section{(A)}

(i)

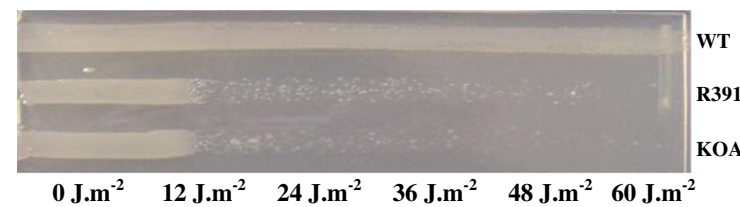

(ii)

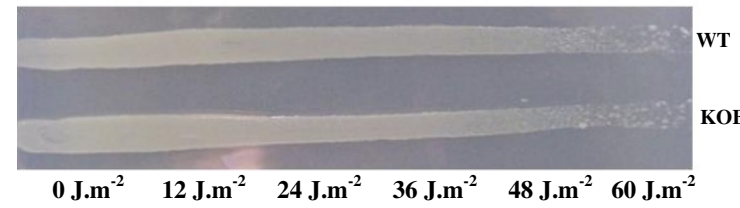

(iii)

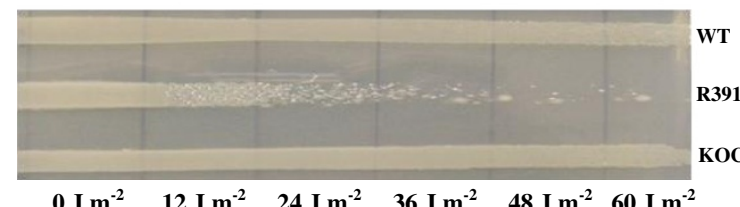

(B)

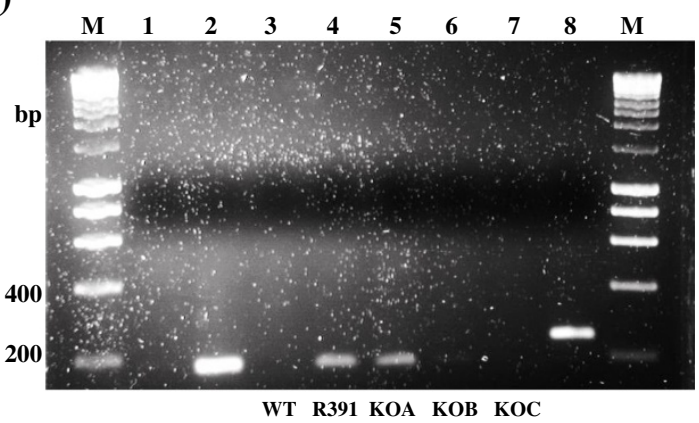

(C)

WT R391 KOA KOB KOC

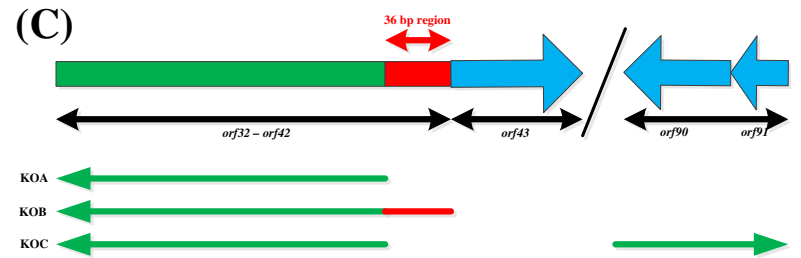

Figure 4 Qualitative UV assay and mRNA analysis of E. coli R391 mutants KOA, KOB and KOC. (A) AB1157 R391 mutants KOA, KOB and KOC. $U_{254 n m}$ exposure increasing (12 J.m²) from left to right. (i) From top to bottom, AB1157, AB1 157 R391, AB1157 R391 KOA. (ii) AB1 157, AB1157 R391 KOB. (iii) AB1157, AB1157 R391, AB1157 R391 KOC. (B) SYBR ${ }^{\oplus}$ Safe stained 1\% (w/v) agarose gel confirming orf43 mRNA transcription in AB1157 R391 KOA. M, Bioline Hyperladder I DNA marker; 1, AB1157 R391 RNA negative control; 2, AB1157 R391 genomic DNA positive control; 3, AB1157 orf43 CDNA; 4, AB1157 R391 orf43 CDNA; 5, KOA orf43 cDNA; 6, KOB orf43 CDNA; 7, KOC orf43 CDNA; 8, KOB orf20 cDNA. Primers used specific to orf43 generated a 188 bp PCR product. Primers used for lane 8 only were specific for the kanamycin resistance gene of ICE R391, orf20, which generated a PCR product of $223 \mathrm{bp}$. Amplification of orf20 specific CDNA was carried out to show KOB and KOC RNA was not degraded. Lane 1 negative control was DNase treated RNA that was not converted to CDNA. (C) Map of exact locations of KOA, KOB and KOC deletions on ICE R391 genome. The KOA, KOB and KOC ampicillin resistance cassettes and associated promoter were inserted into the ICE R391 genome in the reverse complement to prevent the ampicillin resistance cassette promoter inducing the transcription of orf $43 \mathrm{mRNA}$. The KOA deletion removed all possible promoters of orf 43 in front of the gene and left the last $36 \mathrm{bp}$ specific to the preceding orf42 gene. The KOB deletion removed the same region as KOA and the $36 \mathrm{bp}$ region. The $\mathrm{KOC}$ deletion was a duplicate of $\mathrm{KOA}$ with an additional zeocin resistant orfs90/91 deletion.

(A)

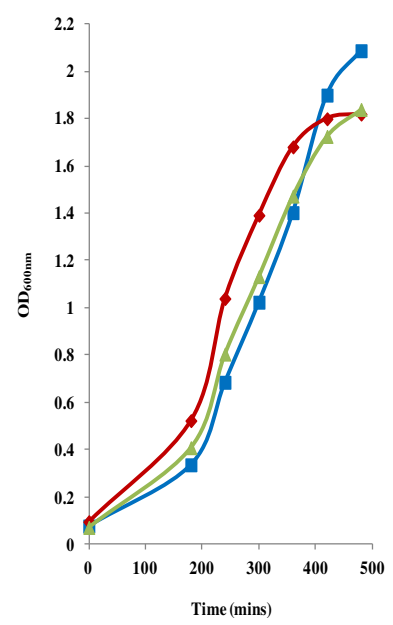

(B)

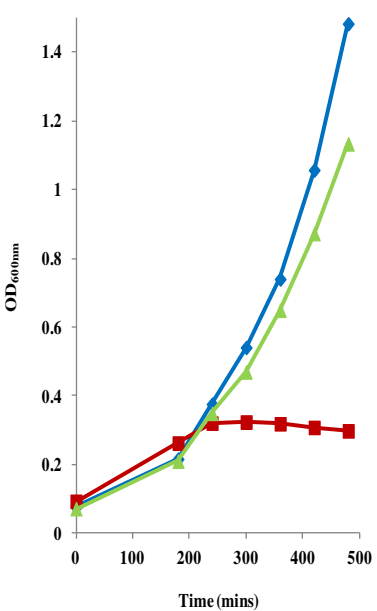

(C)

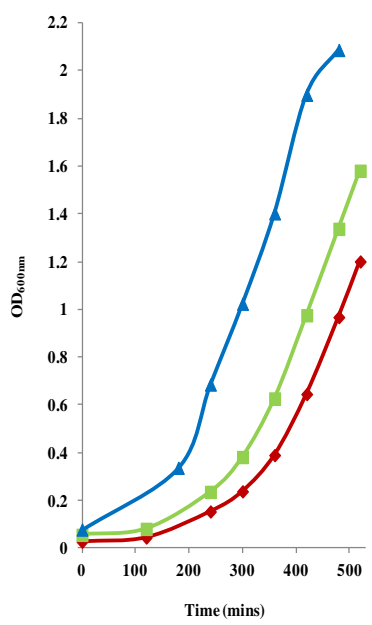

(D)

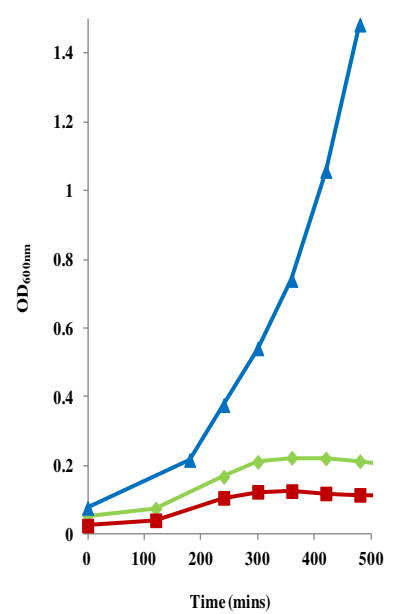

Figure 5 Effect on growth rates of the pBAD33-orf43 SM12 and SM56 mutations in E. coli TOP10. (A) Un-induced growth rates for pBAD33 (blue curve), pBAD33-orf43 (red curve) and pBAD33-orf43 SM12 (green curve). (B) Induced growth rates for pBAD33 (blue curve), pBAD33-orf43 (red curve) and pBAD33-orf43[SM12] (green curve). (C) Un-induced growth rates for pBAD33 (blue curve), pBAD33-orf43 (red curve) and pBAD33-orf43 SM56 (green curve). (D) Induced growth rates for pBAD33 (blue curve), pBAD33-orf43 (red curve) and pBAD33-orf43[SM56] (green curve). Note that the SM12 mutation in pBAD33-orf43 caused a return to exponential growth behaviour expected with E. coli cells. 
formation of the conjugative transfer system of ICE R391, it appears that the associated cytotoxicity is related to the induced overexpression. Evidence in support of this comes from data showing that overexpression of orf 43 from the arabinose inducible clone pBAD33-orf43 leads directly to cytotoxicity [8]. The UV-inducible sensitising effect is conserved amongst many SXT/R391 ICE family members $[6,20]$. A sophisticated control system is in place to control this effect yet the exact nature and reason for conservation of such an unusual apparently 'evolutionary negative' effect remains to be elucidated. We are currently examining the nature of the cytotoxicity and developing theories for its function and retention.

\section{Methods}

\section{Bacterial strains, elements and media}

The bacterial strains, plasmids and ICE R391 deletion mutants utilised as part of this study are listed in Table 1. Strains were stored at $-80^{\circ} \mathrm{C}$ in either Luria-Bertani (LB) broth or M9 minimal media containing 50\% (v/v) glycerol. Media was supplemented with appropriate antimicrobial agents: nalidixic acid, $30 \mu \mathrm{g} \mathrm{ml}^{-1}$; ampicillin, $100 \mu \mathrm{g} \mathrm{ml}^{-1}$; chloramphenicol, $25 \mu \mathrm{g} \mathrm{ml}^{-1}$, kanamycin, $30 \mu \mathrm{g} \mathrm{ml}^{-1}$, streptomycin, $100 \mu \mathrm{g} \mathrm{ml}^{-1}$; mercuric chloride, $20 \mu \mathrm{g} \mathrm{ml}^{-1}$; zeocin, $25 \mu \mathrm{g} \mathrm{ml}^{-1}$ as required. For growth and analysis of strains containing pBAD33-orf43, M9 minimal media containing $0.4 \%(\mathrm{v} / \mathrm{v})$ glycerol was used with either $0.4 \%(\mathrm{w} / \mathrm{v})$ glucose or $0.02 \%-0.2 \%(\mathrm{w} / \mathrm{v})$ L-arabinose to repress or induce gene expression respectively as previously described [8].

\section{Directed deletions of ICE R391 and subsequent deletion mutant screening}

ICE R391 specific deletions were generated as previously described [8]. Screening of resulting ICE R391 deletion mutants for loss of cell-sensitising function by qualitative and quantitative UV survival assays were carried out as described [8]. Screening of ICE R391 deletion mutants' conjugative transfer ability to recipient Salmonella enterica serotype Enteritidis strain P125109 was performed as described [21].

\section{Qualitative reverse transcriptase PCR}

Cells were collected by centrifugation, washed twice with diethyl pyrocarbonate-treated distilled water and resuspended in $10 \mathrm{mM}$ Tris, [pH8.0]. Total RNA was isolated using the Absolutely RNA Miniprep kit (Agilent Technologies) according to the manufacturer's protocol. Absence of contaminating DNA was verified by PCR. Qualitative reverse transcriptase PCR was performed using the AccuScript High Fidelity $1^{\text {st }}$ Strand cDNA Synthesis Kit (Agilent Technologies) according to the manufacturer's protocol. Resulting cDNA was analysed immediately by PCR using gene-specific primers or stored at $-20^{\circ} \mathrm{C}$.

\section{Quantitative reverse transcriptase PCR (qRT-PCR)}

Quantitative UV assays were carried out as described [8]. Unirradiated and irradiated cells were collected by centrifugation and total RNA isolated as described. Absence of contaminating DNA was verified by PCR. qRTPCR was performed using the Brilliant III Ultra-Fast SYBR Green qRT-PCR Master Mix (Agilent Technologies) according to the manufacturer's protocol using the Stratagene Mx3000P Real Time PCR System and appropriate gene-specific PCR primers with the following temperature profile: 1 cycle at $42^{\circ} \mathrm{C}$ for 30 minutes to convert RNA to cDNA, 1 denaturation cycle at $95^{\circ} \mathrm{C}$ for 3 minutes followed by 40 cycles at $95^{\circ} \mathrm{C}$ for 30 seconds, $54^{\circ} \mathrm{C}$ for 60 seconds, $72^{\circ} \mathrm{C}$ for 30 seconds followed by melting curve analysis from $65^{\circ} \mathrm{C}$ to $95^{\circ} \mathrm{C}$ to determine specificity of the PCR reaction. Specificity of the PCR reaction was verified by SYBR safe staining on a $2 \%(\mathrm{w} / \mathrm{v})$ agarose gel. The internal standard curve using the unirradiated RNA sample to estimate the change in target RNA quantity consisted of: undiluted RNA, a 1 in 2 dilution, a 1 in 4 dilution and a 1 in 10 dilution of unirradiated RNA. A no template negative control was also included. In addition, qRT-PCR was also carried out on the known endogenous housekeeping gene proC as an internal control to quantify the relative change in transcription of the gene of interest [22].

\section{Site-directed mutagenesis of pBAD33-orf43}

Site-directed mutagenesis of pBAD33-orf43 [8] was performed using specifically designed complementary mutagenic primers to linearly amplify pBAD33-orf43 to generate a mutated nicked DNA product. Non-mutated methylated template DNA was eliminated by incubation with the DpnI restriction enzyme. Mutated DNA products were then transformed into TOP10 and plated on appropriate media containing chloramphenicol, $25 \mu \mathrm{g} \mathrm{ml} \mathrm{m}^{-1}$. Resulting TOP10 colonies were cultured, had plasmid content extracted using the QIAprep Spin Miniprep Plasmid extraction kit from QIAGEN (West Sussex, RH10, 9NQ, UK) according to the manufacturer's protocol and screened for the presence of pBAD33-orf43 by restriction enzyme digestion. Mutated pBAD33-orf43 was verified by DNA sequencing to contain the desired mutation without additional mutations. Mutated pBAD33-orf43 was confirmed to still transcribe orf 43 specific mRNA by RT-PCR as described. Determination of the effect of induction of mutated pBAD33-orf43 on host cell growth rate was carried out as described [8].

\section{Competing interests}

The authors declare that they have no competing interests. 


\section{Authors' contributions}

PA and JTP conceived and designed the study. PA did the laboratory work and analysed the data. PA and JTP wrote the manuscript. Both authors read and approved the final manuscript.

\section{Acknowledgements}

This work was funded by the Irish Research Council for Science, Engineering and Technology (IRSCET) to PA. The authors would like to thank Dr. P. Latour-Lambert for providing the PKOBEG plasmids and Drs. John O'Halloran and Michael P. Ryan for helpful discussion.

Received: 8 April 2013 Accepted: 28 August 2013

Published: 29 August 2013

\section{References}

1. Taviani E, Grim CJ, Chun J, Huq A, Colwell RR: Genomic analysis of a novel integrative conjugative element in Vibrio cholerae. FEBS Lett 2009, 583(22):3630-3636

2. Michael GB, Kadlec K, Sweeney MT, Brzuszkiewicz E, Liesegang H, Daniel R, Murray RW, Watts JL, Schwarz S: ICEPmu1, an integrative conjugative element (ICE) of Pasteurella multocida: structure and transfer. J Antimicrob Chemoth 2012, 67(1):91-100.

3. Pembroke JT, Piterina AV: A novel ICE in the genome of Shewanella putrefaciens W3-18-1: comparison with the SXT/R391 ICE-like elements. FEMS Microbiol Lett 2006, 264(1):80-88.

4. Brochet $M$, Couve E, Glaser P, Guedon G, Payot S: Integrative conjugative elements and related elements are major contributors to the genome diversity of Streptococcus agalactiae. J Bacterio/ 2008, 190(20):6913-6917.

5. te Poele EM, Bolhuis $H$, Dijkhuizen L: Actinomycete integrative and conjugative elements. A van Leeuw J Microb 2008, 94(1):127-143.

6. Pembroke JT, Stevens E: The effect of plasmid R391 and other inc plasmids on the survival of Escherichia coli after UV irradiation. J Gen Microbiol 1984, 130:1839-1844.

7. Wang TCV, deSaintPhalle B, Millman KL, Fowler RG: The ultravioletsensitizing function of plasmid R391 interferes with a late step of postreplication repair in Escherichia coli. Mutat Res-DNA Repair 1996, 362(3):219-226.

8. Armshaw PA, Pembroke JT: Generation and analysis of an ICE R391 deletion library identifies genes involved in the element encoded UVinducible cell-sensitising function. FEMS Micro Lett 2013, 342(1):45-53.

9. Boltner D, MacMahon C, Pembroke JT, Strike P, Osborn AM: R391: a conjugative integrating mosaic comprised of phage, plasmid, and transposon elements. J Bacteriol 2002, 184(18):5158-5169.

10. Craig NL, Roberts JW: Function of nucleoside triphosphate and polynucleotide in Escherichia coli recA protein-directed cleavage of phage-lambda repressor. J Bio/ Chem 1981, 256(15):8039-8044.

11. Karu AE, Belk ED: Induction of Escherichia coli RecA protein via recBC and alternate pathways - quantitation by enzyme-linked immunosorbentassay (ELISA). Mol Gen Genet 1982, 185(2):275-282.

12. Janion C: Inducible SOS Response System of DNA Repair and Mutagenesis in Escherichia coli. Int J Bio/ Sci 2008, 4(6):338-344.

13. Persky NS, Lovett ST: Mechanisms of Recombination: Lessons from E. coli. Crit Rev Biochem Mol 2008, 43(6):347-370.

14. O'Halloran JA, McGrath BM, Pembroke JT: The orf4 gene of the enterobacterial ICE, R391, encodes a novel UV-inducible recombination directionality factor, Jef, involved in excision and transfer of the ICE. FEMS Microbiol Lett 2007, 272(1):99-105.

15. Fronzes R, Schafer E, Wang LC, Saibil HR, Orlova EV, Waksman G: Structure of a type IV secretion system core complex. Science 2009, 323(5911):266-268

16. O'Reilly EK, Kreuzer KN: Isolation of SOS constitutive mutants of Escherichia coli. J Bacteriol 2004, 186(21):7149-7160.

17. Beaber JW, Hochhut B, Waldor MK: SOS response promotes horizontal dissemination of antibiotic resistance genes. Nature 2004 427(6969):72-74

18. de Henestrosa AR F, Ogi T, Aoyagi S, Chafin D, Hayes JJ, Ohmori $H_{\text {, }}$ Woodgate R: Identification of additional genes belonging to the LexA regulon in Escherichia coli. Mol Microbio/ 2000, 35(6):1560-1572.

19. Nilsson I, Saaf A, Whitley P, Gafvelin G, Waller C, von Heijne G: Prolineinduced disruption of a transmembrane alpha-helix in its natural environment. J Mol Biol 1998, 284(4):1165-1175.
20. McGrath BM, O'Halloran JA, Piterina AV, Pembroke JT: Molecular tools to detect the IncJ elements: a family of integrating, antibiotic resistant mobile genetic elements. J Microbiol Meth 2006, 66(1):32-42.

21. McGrath BM, O'Halloran JA, Pembroke JT: Pre-exposure to UV irradiation increases the transfer frequency of the IncJ conjugative transposon-like elements R391, R392, R705, R706 R997 and pMERPH and is recA(+) dependent. FEMS Microbiol Lett 2005, 243(2):461-465.

22. Theis T, Skurray RA, Brown MH: Identification of suitable internal controls to study expression of a Staphylococcus aureus multidrug resistance system by quantitative real-time PCR. J Microbiol Meth 2007, 70(2):355-362.

doi:10.1186/1471-2180-13-195

Cite this article as: Armshaw and Pembroke: Control of expression of the ICE R391 encoded UV-inducible cell-sensitising function. BMC Microbiology 2013 13:195.

\section{Submit your next manuscript to BioMed Central and take full advantage of:}

- Convenient online submission

- Thorough peer review

- No space constraints or color figure charges

- Immediate publication on acceptance

- Inclusion in PubMed, CAS, Scopus and Google Scholar

- Research which is freely available for redistribution 